\title{
High frequency stimulation for verifying durable lesions during atrial fibrillation ablation.
}

\author{
Kazuo Kato ${ }^{1}$, Shin Hasegawa ${ }^{1}$, Shun Kikuchi ${ }^{1}$, Yukihiro Uehara ${ }^{1}$, Nobuo Ishiguro ${ }^{1}$, \\ Shimpei Tominaga ${ }^{1}$, Akimitsu Tanaka ${ }^{1}$, Ryosuke Kametani ${ }^{1}$, and Norihisa Shibata ${ }^{1}$ \\ ${ }^{1}$ Nagoya Tokushukai General Hospital
}

July 21, 2020

\begin{abstract}
Introduction Exit block, which is difficult to strictly confirm and cannot be adopted for adenosine triphosphate (ATP) injections to provoke dormancy, is an essential concept of atrial fibrillation (AF) ablation. We investigated exit conduction using high frequency stimulation (HFS) and evaluated whether dormant "exit" conduction of the pulmonary veins (PVs) and left atrial posterior wall (LAPW) would remain in patients in which an encircling isolation of all PVs and LAPW isolation (Box PVI) were performed. Methods We enrolled 345 consecutive patients with various severities of AF undergoing a Box PVI and defined the procedure endpoint as complete bidirectional block with no dormant "entrance and exit" conduction of all PVs and the LAPW using HFS and ATP. Results Dormant "exit" conduction of the PVs with HFS delivered at the PV carina following an ATP injection remained in $0.9 \%$ after additional applications, and that for the LAPW was provoked in $5.5 \%$, which remained in $1.4 \%$. Our definition of a complete bidirectional Box PVI was satisfied in $79.7 \%$ and showed the best clinical outcome. In the non-paroxysmal AF group, there were significant differences in the recurrence rates between the groups with and without a complete LAPW isolation, however, the clinical outcome was independent of a complete LAPW isolation in the paroxysmal AF group. Conclusion HFS delivered at the PV carina and LAPW following a Box PVI could clearly elucidate true exit block, and a concomitant ATP injection could define dormant "exit" conduction, suggesting incomplete lesions that had been missed.
\end{abstract}

Title: High frequency stimulation for verifying durable lesions during atrial fibrillation ablation.

Authors:

Kazuo Kato, M.D., Ph.D.

Shin Hasegawa, M.D.

Shun Kikuchi, M.D.

Yukihiro Uehara, M.D.

Nobuo Ishiguro, M.D.

Shimpei Tominaga, M.D.

Akimitsu Tanaka, M.D.

Ryosuke Kametani, M.D., Ph.D.

Norihisa Shibata, C.E.

Affiliation:

Department of Cardiology, Nagoya Tokushukai General Hospital, Kasugai, Japan 
address:

2-52, Kozoji-cho kita, Kasugai, Aichi, Japan. 487-0016 phone: +81-568-51-8711Ｆax: +81-568-51-7115

Sources of Funding:

This research did not receive any specific grant from funding agencies in the public, commercial, or not-forprofit sectors.

Disclosures:

None.

Corresponding Author:

Kazuo Kato e-mail:kkato@fujita-hu.ac.jp

Author contributions:

Kazuo Kato performed the conception, design, and data collection. Shin Hasegawa, Shun Kikuchi, Yukihiro Uehara, Shimpei Tominaga, Akimitsu Tanaka, Ryosuke Kametani, and Norihisa Shibata performed the data collection.

\section{$<$ Abstract $>$}

Introduction

Exit block, which is difficult to strictly confirm and cannot be adopted for adenosine triphosphate (ATP) injections to provoke dormancy, is an essential concept of atrial fibrillation (AF) ablation. We investigated exit conduction using high frequency stimulation (HFS) and evaluated whether dormant "exit" conduction of the pulmonary veins (PVs) and left atrial posterior wall (LAPW) would remain in patients in which an encircling isolation of all PVs and LAPW isolation (Box PVI) were performed.

Methods

We enrolled 345 consecutive patients with various severities of AF undergoing a Box PVI and defined the procedure endpoint as complete bidirectional block with no dormant "entrance and exit" conduction of all PVs and the LAPW using HFS and ATP.

Results

Dormant "exit" conduction of the PVs with HFS delivered at the PV carina following an ATP injection remained in $0.9 \%$ after additional applications, and that for the LAPW was provoked in $5.5 \%$, which remained in 1.4\%. Our definition of a complete bidirectional Box PVI was satisfied in $79.7 \%$ and showed the best clinical outcome. In the non-paroxysmal AF group, there were significant differences in the recurrence rates between the groups with and without a complete LAPW isolation, however, the clinical outcome was independent of a complete LAPW isolation in the paroxysmal AF group.

Conclusion

HFS delivered at the PV carina and LAPW following a Box PVI could clearly elucidate true exit block, and a concomitant ATP injection could define dormant "exit" conduction, suggesting incomplete lesions that had been missed.

\section{$<$ Keywords $>$}

Dormant conduction, Adenosine triphosphate, Bidirectional block, Exit block, Box isolation, Pulmonary vein isolation

\section{Introduction:}


Pulmonary vein (PV) isolation (PVI) is a corner stone of atrial fibrillation (AF) treatment. ${ }^{1}$ Recently, a wider PVI has been reported to obtain a better recurrence rate even in patients with paroxysmal $\mathrm{AF},{ }^{2,3}$ and a PVI including the posterior wall of the left atrium (LA) (LAPW), i.e., a box shaped PVI (Box PVI), has also been proposed as a good option for the various types of AF ablation. ${ }^{4}$

The injection of adenosine triphosphate (ATP) is reported to be another option for a better outcome of checking the durability of lesions, by provoking dormant conduction leading to reconnections of the isolation lines and then recurrence of AF. ${ }^{5,6}$ However, dormant conduction elicited under conventional methods is evaluated only regarding the entrance conduction based on the reappearance of PV electrograms, i.e., dormant "entrance" conduction of the PVs.

Since part of the background of the PVI has been based upon the blockade of abnormal PV firing to the LA, ${ }^{7,8}$ it would be important to confirm not only entrance block but also exit block as well with the ATP administration. In this study, we investigated the exit conduction using high frequency stimulation (HFS), and moreover, we evaluated whether dormant "exit" conduction remained on the LAPW in addition to the PVs in patients that underwent a Box PVI in which complete bidirectional block was conventionally confirmed including no dormant "entrance" conduction.

\section{Methods:}

\section{Study population}

Three hundred forty-five consecutive patients with symptomatic drug-refractory paroxysmal, persistent, and long-standing (LS) persistent AF were enrolled in this retrospective cohort study who were referred to our institution for an initial catheter ablation. We defined paroxysmal AF as that terminating spontaneously and lasting for less than 1 week, persistent AF as that lasting for more than 1 week but less than 1 year, and LS persistent AF as that lasting for longer than 1 year. Written informed consent was obtained from all patients. The institutional review board approved the study protocol, and all patients provided written informed consent for the procedure.

\section{Study protocol}

\section{Ablation procedure}

The procedures were performed at least 1 month after the withdrawal of all antiarrhythmic drugs. Amiodarone was not prescribed in any patients. Multidetector computed tomography was performed prior to the procedure to evaluate the anatomical variations in the LA and PVs in each patient. All the procedures were performed under deep sedation with dexmedetomidine, propofol, and buprenorphine, and with esophageal temperature and direct blood pressure monitoring. The activated clotting time was kept at approximately 300 seconds all through the procedure. An irrigation catheter with a contact force sensor through a deflectable sheath in combination with a 3D mapping system (CARTO, Biosense-Webster, Diamond Bar, CA) with the use of at least one circular catheter or PentaRay catheter was used in all patients. We delivered radiofrequency (RF) energy at 30 to 40 Watts on the anterior and roof regions and 20 to 30 Watts on the posterior region of the PVs while measuring the contact force $(\mathrm{CF})$ kept between 5 and $20 \mathrm{~g}$ for all $\mathrm{RF}$ applications. After a standard transseptal puncture, RF energy was applied to the anterior portion of each PV along with the LA roof and bottom regions for a box shaped encircling of all PVs and an LAPW isolation (Box PVI) in all patients. If the electrograms of any PVs or the LAPW remained after creating one circular line, we tried to apply additional RF energy to the gaps on the isolation line or inside the box until they were eliminated.

\section{Feasibility study of using HFS to confirm exit block for a Box PVI}

First of all, in order to elucidate the feasibility of the HFS for verifying exit block, we examined the response to delivering HFS at $20 \mathrm{~Hz}$, with an amplitude of $20 \mathrm{~V}$, and a pulse width of $10 \mathrm{~ms}$, which was the maximum power of the electrical stimulator (SEC-5104, Nihon Kohden, Tokyo, Japan), on the LAPW or inside of the PVs during sinus rhythm (SR) before the ablation while monitoring the CF (Figure 1). The atrial rhythm 
became irregular during the HFS delivery at all sites in the atrium even with only $1 \mathrm{~g}$ of CF in all cases, and we defined that as an "HFS (+)" response (Figure 1A). On the other hand, HFS delivered for more than 5 seconds with more than $5 \mathrm{~g}$ of $\mathrm{CF}$ disrupted the atrial rhythm and provoked AF after ceasing the HFS in all cases, and we defined that as an "HFS (++)" response (Figure 1B). Thus, no cases remained in SR during the HFS delivery before the ablation, which we defined as an "HFS (-)" response. According to the above findings that the HFS delivery could activate the atrial myocardium with a minimum CF in all cases, we thought the HFS would be feasible for evaluating the local conductivity i.e., exit block, and we then examined the completion of the PVI and Box PVI as follows after confirming entrance block with the disappearance of all PV and LAPW electrograms.

\section{Protocol to confirm the completion of the bidirectional isolation of the PVs and LAPW}

First of all, we confirmed the disappearance of the electrograms from each PV and the LAPW inside of the encircling line independently using a circular catheter with 20 electrodes or a PentaRay catheter, i.e., entrance block after delineating the single encircling line around all PVs and the LAPW. We applied additional RF energy to the gap or inside the box area if any electrograms remained. Then we restored the rhythm to SR using cardioversion if the rhythm was not already restored to SR after creating the box lesion in order to check the exit block based upon the regularity of the atrial rhythm.

We defined complete exit block of the ipsilateral PVs as the absence of carina conduction, ${ }^{9,10}$ so we delivered HFS separately on both sides of the carinas and then at the center of the LAPW for more than 7 seconds with more than a $10 \mathrm{~g} \mathrm{CF}$ in order to confirm whether or not the HFS could affect the atrial rhythm, i.e., to confirm whether exit block of each the PVI and the box lesion was completed. If the atrial rhythm was affected during the HFS delivery, with exit conduction still remaining (HFS $(+) /(++)$ ), we added RF energy to the gaps to eliminate the exit conduction to accomplish bidirectional conduction block. Then, after waiting for more than $20 \mathrm{~min}$ after the completion of the bidirectional block, we injected $40 \mathrm{mg}$ of ATP to check whether or not the PV electrograms would appear again, to confirm whether dormant "entrance" conduction of the PV could be provoked. If no dormant "entrance" conduction appeared, we then delivered the HFS with a CF of more than $10 \mathrm{~g}$ at the center of the LAPW for more than 3 seconds just when atrio-ventricular (AV) block was provoked during the ATP injection in order to examine whether the atrial rhythm could be affected by the HFS, to confirm whether dormant "exit" conduction of the box area could be provoked. We defined the endpoint of the procedure as complete bidirectional block with no dormant "entrance or exit" conduction of all PVs and the LAPW, and then we applied additional RF energy, if necessary, to any gaps to eliminate both the dormant "entrance" and "exit" conduction. After that, we finally delivered the HFS with a $\mathrm{CF}$ of more than $10 \mathrm{~g}$ on both carinas for more than 3 seconds during an ATP injection in order to examine whether dormant "exit" conduction of the PV could also be provoked in cases with dormant "exit" conduction of the LAPW.

\section{Patient follow-up}

After the Box PVI procedure, all patients received clinical follow-up at 2 weeks, 1, 3, 6, and 12 months, and at least once a year to check their 12-lead ECG and any symptoms. Antiarrhythmic drugs were continued for the first 3 months after the procedure in some patients with persistent or LS persistent AF and then ceased in all patients after the procedures. A 24-hour Holter monitoring was performed at least 3 times after 3 months and within 12 months in all patients, and an ambulatory ECG recorder (HCG-801, Omron, Kyoto, Japan) was used if the patients felt any unidentified palpitations in order to check if any tachyarrhythmias could be documented. We defined an arrhythmia recurrence in the patients if any atrial tachyarrhythmias lasting more than 30 seconds could be documented.

\section{Statistical Analysis}

Continuous variables are expressed as the mean $\pm \mathrm{SD}$ or median with the first quartile to third quartile [Q1Q3]. We analyzed the parametric data using a Student's t-test and a Wilcoxon analysis and Kruskal-Wallis analysis for the nonparametric data. A Chi-squared analysis was used for the categorical variables. The event-free rates were calculated using the Kaplan-Meier survival method, and log-rank statistics were used 
for comparisons between groups. Data Analyses were performed using JMP version 14.2.0 software (SAS Institute, Cary, NC). A p $<0.05$ was considered statistically significant.

\section{Results}

\section{Baseline Characteristics}

The baseline characteristics of each subgroup of patients are listed in Table 1 . They included 249 men and 96 women with a mean age of $66.2 \pm 10.2$ years. The median follow-up period was 558.0 [287.0-870.5] days. There were some demographic differences among each subgroup.

\section{Response to HFS delivered at the PV carinas and LAPW}

All PV and LAPV electrograms were eliminated in all cases, i.e., we could confirm the conventional entrance block in all cases after creating circular lines to encircle each PV antrum and LAPW and additional RF applications to the gaps or residual electrograms inside the box lesion. Upon applying the HFS to each carina, most of the atrial rhythms were not affected and SR continued with an HFS (-) response (Figure 2A), which meant conventional exit block of the PVs was completed. However, in 15 cases $(4.3 \%)$ the rhythms were affected resulting in an irregular rhythm during the HFS, i.e., an HFS (+) response, even after additional $\mathrm{RF}$ deliveries to the gaps or posterior antrum inside the box in cases it was necessary, however, no cases developed AF, and thus, no cases had an HFS $(++)$ response. Some of the atrial rhythms were affected by the HFS inside the isolated lines where automatic firing was observed (Figure 2B). Furthermore, some others exhibited PV entrance reconnections following the HFS delivery as if the HFS had provoked dormant conduction (not shown). On applying the HFS to the LAPW after creating a single circular line, 106 cases $(30.7 \%)$ exhibited an HFS $(+)$ response, and additional RF deliveries inside the box changed the HFS $(+)$ to an HFS (-) response in 43 of those cases (40.6\%). No cases with an HFS (+) response at any PV carina exhibited an HFS (-) response on the LAPW.

More than 20 minutes after the above examinations of the HFS, 59 cases (17.1\%) exhibited dormant "entrance" conduction provoked by ATP or a very early recurrence of PV electrograms. All of the cases with positive dormant "entrance" PV conduction exhibited positive responses to the HFS on the LAPW, and the dormant "exit" conduction remained at the PV carina in 3 cases $(0.9 \%)$ after additional RF applications. We evaluated the LAPW reconnections by means of the existence of dormant "exit" conduction (Figure 3A, B). Dormant "exit" conduction on the LAPW was provoked in $19(5.5 \%)$ cases, and we could abolish it in 14 cases $(73.7 \%)$ out of those cases after additional RF applications.

Both dormant "entrance" PV conduction and dormant "exit" LAPW conduction were negative in 275 $(79.7 \%)$ cases, and the bidirectional Box PVI could be completed. There was no significant difference in the procedural result rates among the subgroups classified by the AF severity, and we encountered no procedure related complications (Figure 4, and Table 2).

\section{Clinical outcome following the Box PVI confirmed by HFS}

The AF recurrence-free rate differed among the groups classified based upon the HFS response and AF severity. The cases in which the Box PVI was completed had a similar recurrence rate independent of their AF severity (Figure 5A). However, cases in which the PVI was completed only in the persistent AF and LS persistent AF groups, which were the cases without a complete LAPW isolation, tended to have a worse prognosis than those in the paroxysmal AF group (Figure 5B).

In order to compare the clinical outcome according to the AF severity, we combined the persistent $\mathrm{AF}$ and LS persistent AF groups to define the non-paroxysmal AF group. In the non-paroxysmal AF group, the best clinical outcome was obtained in patients with a complete bidirectional Box PVI, and there were significant differences between the groups with and without a complete LAPW isolation (log-rank test, $\mathrm{P}=0.0240$ ) (Figure 5C). On the other hand, in the paroxysmal AF group, there were no significant differences between the groups with and without a complete LAPW isolation (Figure 5D).

\section{Discussion}


A durable PVI, which is important to obtain a better outcome of AF ablation, could be confirmed by both entrance and exit block, exhibiting bidirectional conduction block between the PVs and LA, was defined by the disappearance of the PV electrograms and the loss of atrial capture with PV stimulation. ${ }^{8,11}$ Since the PVI has been partially based on the blockade of abnormal PV firing to the LA, it would be important to confirm not only the entrance block but also the exit block. ${ }^{8,11}$ However, the recent guidelines indicate entrance block as the primary endpoint for class I and exit block for class IIb with a lower evidence level. ${ }^{8}$ Furthermore, dormant conduction using an ATP injection to predict insufficient lesions can be evaluated based on only unidirectional entrance conduction, ${ }^{5,6}$ and has hardly been performed for the exit conduction. ${ }^{12}$

Recently, a wider PVI such as a Box PVI has been proposed as a good option for AF ablation, especially of non-paroxysmal AF. ${ }^{4,13}$ Some of those cases have a large LA with tiny PV potentials. The disappearance of the local electrograms could be required for the confirmation of entrance block, however, it might be difficult to evaluate the local potentials of the PVs and LAPW if they are too tiny. Furthermore, when under an ATP injection, in some cases the dormant "entrance" conduction could also be missed, and moreover, some local electrograms from the LAPW could be tinier and hardly recordable in a large LA as compared to the PV electrograms, so it might be far more difficult to evaluate dormant "entrance" conduction on the LAPW in patients undergoing a Box PVI.

In this study, we delivered the HFS to examine the exit conduction after confirming conventional entrance block of the PVI, and then evaluated the dormant "exit" conduction of the LAPW following the Box PVI on an ATP administration using HFS as well. Since the HFS of the heart is well known to provoke fibrillation, ${ }^{14-16}$ and our feasibility study also demonstrated that HFS in the LA with a trivial CF affected the SR, we did not need to confirm local capture when evaluating the exit block using HFS, which also enabled evaluating dormant "exit" conduction on an ATP administration and assured durable lesions with bidirectional complete block of the very tiny potential areas such as the LAPW in LS persistent AF cases. Furthermore, the HFS on the carinas and LAPW using the ablation catheter could hardly capture tissue other than the encircling lesion, which would prevent far-field capture and pseudo-exit conduction. ${ }^{17,18}$ Further, dormant "exit" conduction other than dormant "entrance" conduction might be detected in spite of the potential presence of a source-sink mismatch. ${ }^{19}$ Moreover, some cases, who had non-conducted automatic firing from the PVs after the PVI that meant complete exit block, ${ }^{7,8}$ demonstrated an HFS $(+)$ response, i.e., incomplete exit block (Figure $2 \mathrm{~B}$ ).

According to our results with the use of HFS, the best clinical outcome was obtained in the group in which a complete bidirectional Box PVI was confirmed, and there was a significant difference in the non-paroxysmal AF group between those with and without bidirectional block of the LAPW, which was reasonably compatible with the previous reports. ${ }^{17}$ We encountered a different kind of atrial response to the HFS depending on the $\mathrm{CF}$ where there were no isolation lines before the ablation, namely, HFS $(+)$ and HFS $(++)$ responses, in the feasibility study. On the other hand, no cases had an HFS (++) response with an ordinary CF but some did have an HFS $(+)$ response after an ablation with entrance block. There should be some difference between an HFS (++) and HFS $(+)$ response, however, both of them suggest incomplete block, and we think that HFS is a feasible tool for examining the conductivity of the isolation line for AF ablation. Unfortunately, HFS could not give us any hint of where to additionally ablate, however, that suggested there could be incomplete lesions anywhere.

\section{Limitations}

There were some limitations. First, we delivered the HFS for 3 seconds to check for dormant "exit" conduction soon after the AV block was provoked after confirming no dormant "entrance" conduction with the ATP injection. It could be possible that the dormant "entrance" conduction could have also occurred after the AVB during the HFS delivery, which meant that it might not have been sufficient to consider that only dormant "exit" conduction had occurred. However, we injected ATP to examine the dormant conduction more than 20 minutes after the final RF application, which suggested the potential discrepancy in the response between the entrance and exit might have been very small. All of the cases with dormant "entrance" conduction were revealed to have dormant "exit" conduction, but all of those cases with dormant "exit" conduction did not 
have dormant "entrance" conduction. We thought that dormant "exit" conduction provoked during an ATP injection with HFS would suggest specific unrevealed insufficient lesions. Moreover, it might be too short a duration to evaluate the dormant "exit" conduction by means of an HFS delivery for just 3 seconds. However, a longer HFS duration would delay the backup pacing, which is required in order to avoid prolonged AV block. A longer HFS delivery could induce more dormant "exit" conduction, or we might have missed latent cases with dormant "exit" conduction.

Second, to avoid the phrenic nerve, right atrium, and ventricular capture, we delivered the HFS on the posterior side rather than the anterior side of the carinas to evaluate the exit block. Therefore, we could have underestimated the complete exit block especially on the anterior side of the Box PVI.

Finally, it would be better to determine the clinical significance of the dormant "exit" conduction regarding the freedom from AF recurrence. However, we intended to abolish the dormant "exit" conduction, so that the cases with the dormant "exit" conduction received further RF applications. From an ethical point of view, we could not compare the prognosis of the cases with and without "pure" dormant "exit" conduction. Furthermore, it would not be adequate for a strict evaluation of the recurrence rate to use 24-hour Holter ECG and ambulatory ECG monitoring. However, we finally classified the cases into 2 groups based upon our results and obtained a reasonable outcome. We thought our methods were appropriate.

\section{Conclusion}

HFS delivered at the PV carina and LAPW following a Box PVI could clearly elucidate true exit block, and a concomitant ATP injection could define the dormant "exit" conduction, suggesting incomplete lesions that had been missed. HFS may provide new insight for confirming exit conduction that had been underestimated until now.

Acknowledgments:

The authors thank Mr. John Martin for his grammatical assistance and Mr. Makoto Utsugi for his statistical advice.

Reference

1. Haïssaguerre M, Jaïs P, Shah DC, Takahashi A, Hocini M, Quiniou G, et al. Spontaneous initiation of atrial fibrillation by ectopic beats originating in the pulmonary veins. N Engl J Med 1998; 339: 659-666.

2. Pappone C, Santinelli V, Manguso F, Vicedomini G, Gugliotta F, Augello G, et al. Pulmonary vein denervation enhances long-term benefit after circumferential ablation for paroxysmal atrial fibrillation. Circulation 2004; 109: 327-334.

3. Kiuchi K, Kircher S, Watanabe N, Gaspar T, Rolf S, Arya A, et al. Quantitative analysis of isolation area and rhythm outcome in patients with paroxysmal atrial fibrillation after circumferential pulmonary vein antrum isolation using the pace-and-ablate technique. Circ Arrhythmia Electrophysiol 2012; 5: 667-675.

4. Kumagai K, Muraoka S, Mitsutake C, Takashima H, Nakashima H. A New Approach for Complete isolation of the posterior left atrium including pulmonary veins for atrial fibrillation. J Cardiovasc Electrophysiol 2007; 18: 1047-1052. 5. Arentz T, Macle L, Kalusche D, Hocini M, Jais P, Shah D, et al. "Dormant" pulmonary vein conduction revealed by adenosine after ostial radiofrequency catheter ablation. J Cardiovasc Electrophysiol 2004; 15: 1041-1047. 6. Macle L, Khairy P, Weerasooriya R, Novak P, Verma A, Willems $\mathrm{S}$, et al. Adenosine-guided pulmonary vein isolation for the treatment of paroxysmal atrial fi brillation: an international, multicentre, randomised superiority trial. Lancet 2015; 386: 672-679.

7. Chen S, Meng W, Sheng He D, Chen G, Zhang F, Yan Y, et al. Blocking the pulmonary vein to left atrium conduction in addition to the entrance block enhances clinical efficacy in atrial fibrillation ablation. PACE - Pacing Clin Electrophysiol 2012; 35: 524-531.

8. Calkins H, Hindricks G, Cappato R, Kim Y, Saad E, Aguinaga L, et al. 2017 
HRS/EHRA/ECAS/APHRS/SOLAECE Expert consensus statement on catheter and surgical ablation of atrial fibrillation. Europace 2018; 20: e1-e160.

9. Cabrera JA, Ho SY, Climent V, Fuertes B, Murillo M, Sánchez-Quintana D. Morphological evidence of muscular connections between contiguous pulmonary venous orifices: Relevance of the interpulmonary isthmus for catheter ablation in atrial fibrillation. Heart Rhythm 2009; 6: 1192-1198.

10. Takigawa M, Yamada T, Yoshida Y, Ishikawa K, Aoyama Y, Yamamoto T, et al. The incidence and clinical significance of non-isolation of the pulmonary vein carina after encircling ipsilateral pulmonary veins isolation for paroxysmal atrial fibrillation: A pitfall of the double-Lasso technique. Europace 2013; 15: 33-40.

11. Ouyang F, Bänsch D, Ernst S, Schaumann A, Hachiya H, Chen M, et al. Complete isolation of left atrium surrounding the pulmonary veins: New insights from the double-lasso technique in paroxysmal atrial fibrillation. Circulation 2004; 110: 2090-2096.

12. Spera FR, Heidbuchel H, Sarkozy A, Webster B. Dormant conduction: It is time to wake up. J Am Coll Cardiol EP 2020; 5: 2019-2021.

13. Kim J, Shin S, Na J, Choi C, Kim S, Kim J, et al. Does isolation of the left atrial posterior wall improve clinical outcomes after radiofrequency catheter ablation for persistent atrial fibrillation? A prospective randomized clinical trial. Int J Cardiol 2015; 181: 277-283.

14. Scherlag BJ, Yamanashi WS, Schauerte P, Scherlag M, Sun Y, Hou Y, et al. Endovascular stimulation within the left pulmonary artery to induce slowing of heart rate and paroxysmal atrial fibrillation. Cardiovascular Research 2002; 54: 470-475.

15. Ishibashi K, Inoue D, Sakai R, et al. Effects of disopyramide on the atrial fibrillation threshold in the human atrium. Int J Cardiol 1995; 52: 177-184.

16. Voroshilovsky O, Qu Z, Lee MH, Ohara T, Fishbein GA, Huang HLA, et al. Mechanisms of ventricular fibrillation induction by $60-\mathrm{Hz}$ alternating current in isolated swine right ventricle. Circulation 2000; 102: 1569-1574.

17. Vijayaraman P, Dandamudi G, Naperkowski A, Oren J, Storm R, Ellenbogen KA. Assessment of exit block following pulmonary vein isolation: Far-field capture masquerading as entrance without exit block. Heart Rhythm 2012; 9: 1653-1659.

18. Ip JE, Markowitz SM, Cheung JW, Liu CF, Thomas G, Lessner SJ. Method for differentiating left superior pulmonary vein exit conduction from pseudo-exit conduction. PACE - Pacing Clin Electrophysiol 2013; 36: 299-308.

19. Spector P: Principles of cardiac electric propagation and their implications for re-entrant arrhythmias. Circ Arrhythmia Electrophysiol 2013; 6: 655-661.

Figure legends

Figure 1:

Intracardiac tracings recorded during high frequency stimulation (HFS) on the left atrial posterior wall (LAPW) during sinus rhythm (SR) before the ablation with a contact force (CF) of 1-2 g (A) and $9 \mathrm{~g}(\mathrm{~B})$. A: The atrial rhythm became irregular during the HFS, and we defined that as an HFS (+) response. B: The HFS disrupted the atrial rhythm provoking AF after cessation of the HFS, and we defined that as an HFS $(++)$ response. The tracings are surface ECG leads I, aVF, V1, V5, and the intracardiac electrograms recorded by the distal to proximal electrodes of a high right atrial (HRAd-p) catheter, distal to proximal electrograms recorded by a circular catheter within the pulmonary vein (PVd-p), distal, proximal, and unipolar electrograms recorded by an ablation catheter (ABLd, ABLp, and ABLu) on the LAPW, distal to proximal coronary sinus catheter (CSd-p) electrograms, and the blood pressure.

Figure 2: 
Intracardiac tracings recorded during HFS on the LAPW during SR after a complete box pulmonary vein isolation (Box PVI) (A) and incomplete Box PVI (B). A: Bump artifact of the catheter on the LAPW (asterisks) that preceded the PV electrograms did not conduct to the atrium, which certified that the catheter was in contact with the LAPW, and the atrial rhythm was not affected during or after the HFS. We defined this tracing manner as an HFS (-) response. B: The atrial rhythm was affected during the HFS, however, a preceding non-conducted automatic firing was observed, which was an HFS $(+)$ response. The order of the tracings and abbreviations are the same as in Figure 1.

Figure 3:

Intracardiac tracings recorded during HFS on the LAPW in SR while provoking atrio-ventricular block by an adenosine triphosphate administration after a Box PVI in cases that underwent a complete Box PVI (A) and incomplete Box PVI (B). A: The atrial rhythm was not affected by the HFS, which was an HFS (-) response. Note that the bump artifact of the catheter on the LAPW was similar to that in Figure 2A, which certified that the catheter was in contact with the LAPW. B: The atrial rhythm was affected by the HFS, which was an HFS (+) response. However, automatic firing before and after the HFS was observed. The order of the tracings and abbreviations are the same as in Figures 1 and 2.

Figure 4: Flowchart of the procedure.

Conventional entrance block of the box lesion was obtained in all study subjects, however, HFS and a concomitant adenosine triphosphate (ATP) injection could elicit incomplete lesions in some cases. See the text and Table 2 for the details. The abbreviations are the same as in Figures 1, 2, and 3. LAPWI, left atrial posterior wall isolation; RF, radiofrequency.

Figure 5:

Kaplan-Meier curves of the comparison of the freedom from atrial fibrillation (AF) after a box pulmonary vein isolation (Box PVI) among the different severities of $\mathrm{AF}$ in patients that underwent a complete Box PVI (A), those that underwent a complete PVI without a complete left atrial posterior wall isolation (B), between a complete Box PVI and incomplete Box PVI in patients with non-paroxysmal AF (C), and those with paroxysmal AF (D). The abbreviations are the same as in Figures 1, 2, and 3. LS, long-standing. 

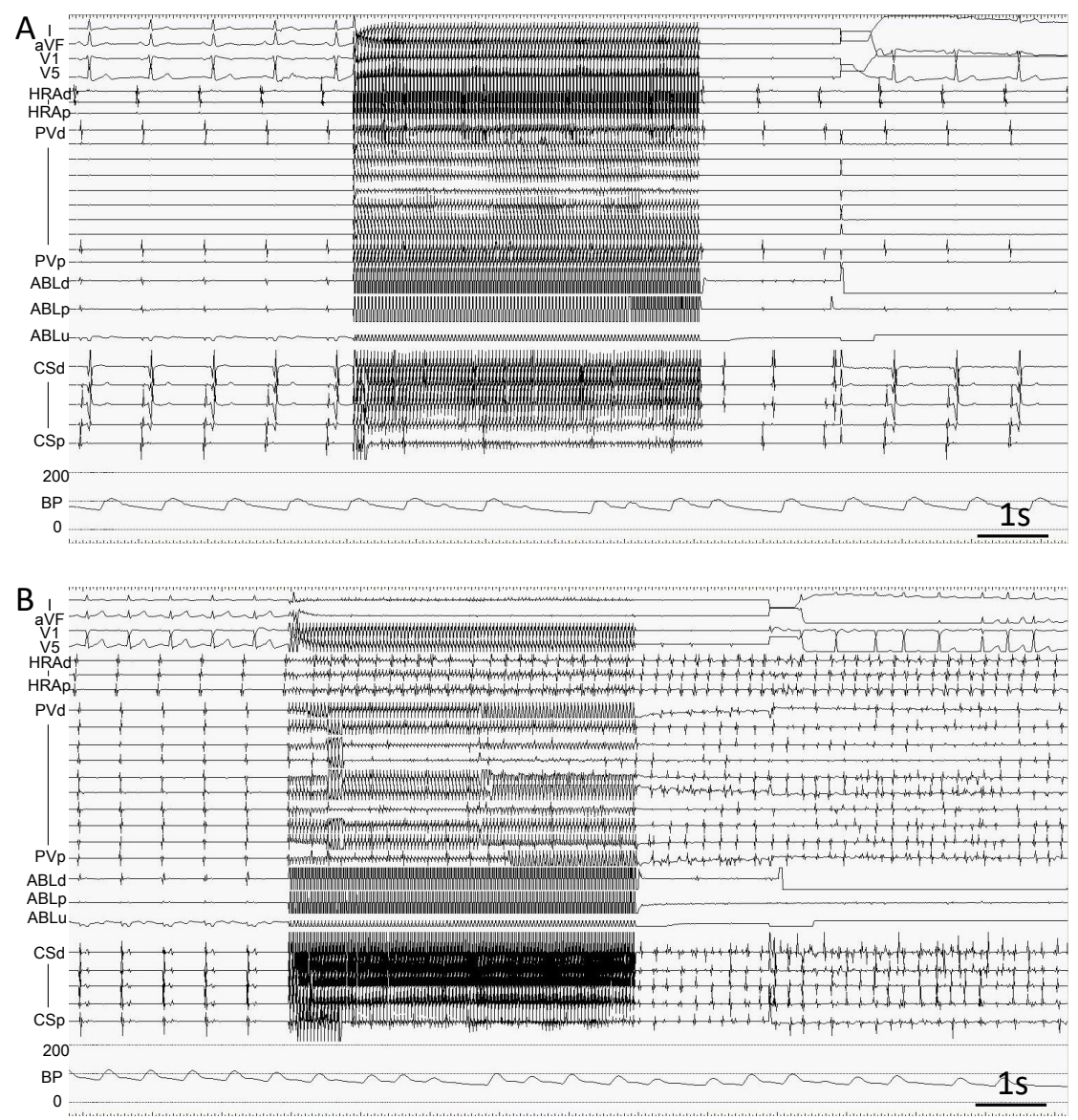

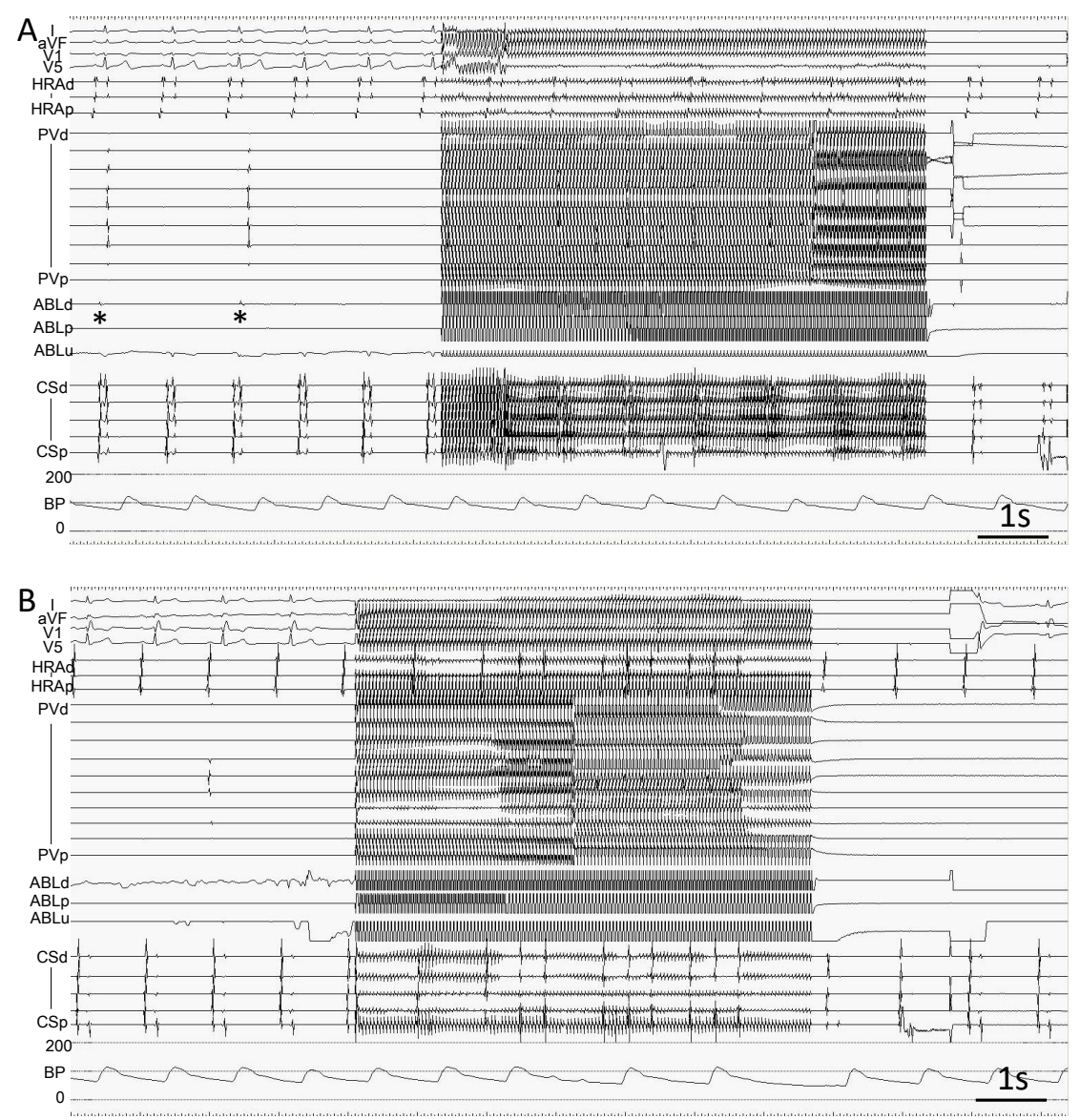

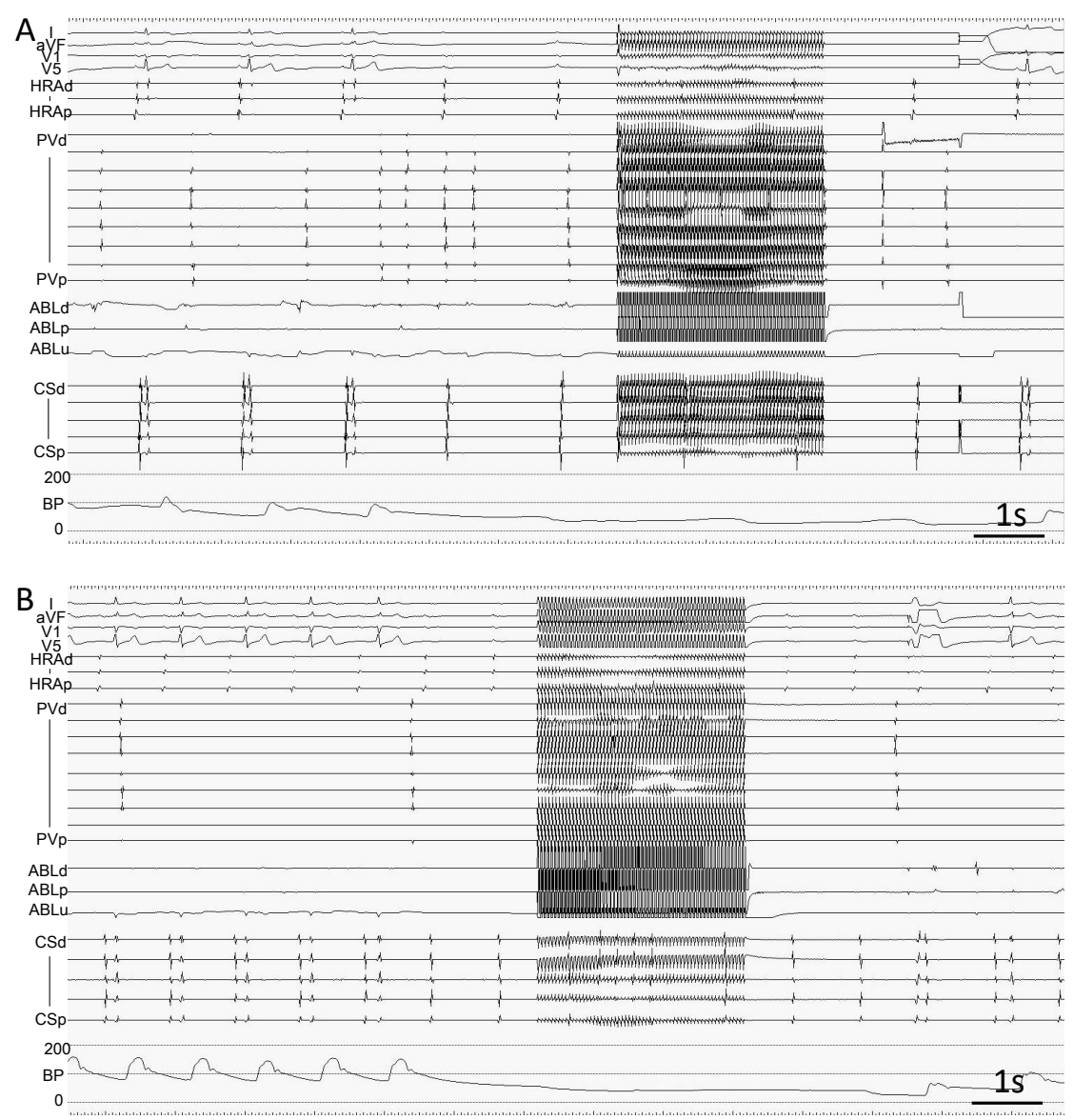

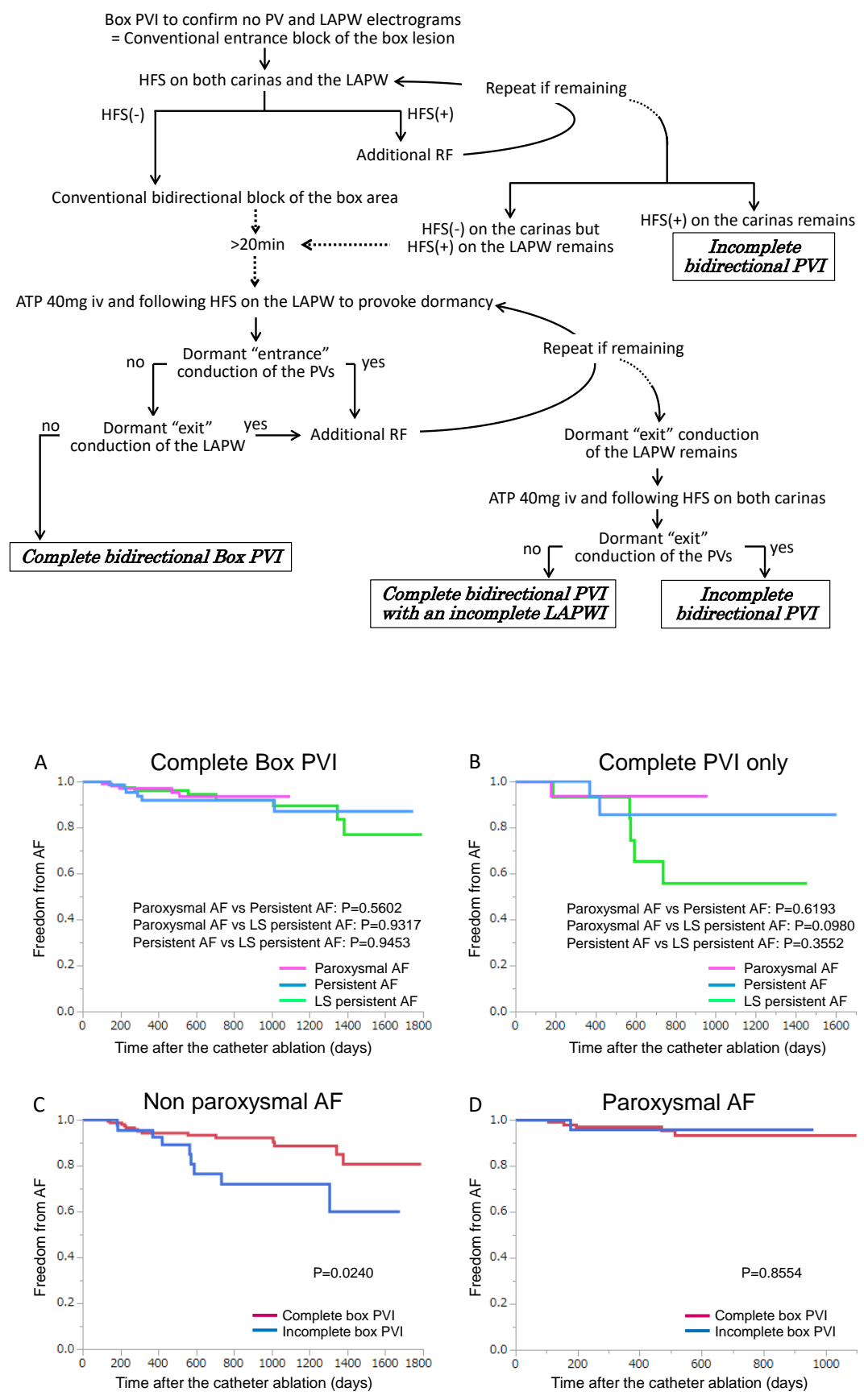

\section{Hosted file}

JCEHFStable1KK.docx available at https://authorea.com/users/344612/articles/471066-highfrequency-stimulation-for-verifying-durable-lesions-during-atrial-fibrillation-ablation

\section{Hosted file}

JCEHFStable2KK.docx available at https://authorea.com/users/344612/articles/471066-highfrequency-stimulation-for-verifying-durable-lesions-during-atrial-fibrillation-ablation 